\title{
Integrating genomics into US public health
}

Allan S. Noonan, MD, MPH

This paper presents an overview of how the US government is currently addressing the complex issue of integrating
genomics into public health. The author, a public health provider with many years of experience, including several
years spent in genetics and maternal and child health, is currently a senior advisor to the Surgeon General of the
United States. At the time of the third biennial Asan-Harvard Medical International Symposium, "Genomics and
Proteomics: Impact on Medicine and Health," in Seoul, Korea, July 3-4, 2001, the US Surgeon General was Dr.
David Satcher. Dr. Allan Noonan attended the symposium as Dr. Satcher's representative. Dr. Noonan's paper
discusses the challenges facing the US public health system as it strives to integrate and promote genomics and
proteomics into its efforts to reduce morbidity and mortality in the US population. Genet Med 2002:4(6, Supplement):68S-71S.

Key Words: genomics, proteomics, public health, US government

The integration of genomics into public health is a complex issue. But before embarking on a discussion of the issue, let us remind ourselves of the major functions of public health, based on the Institute of Medicine definition of public health, namely, assessment, policy development, and assurance. ${ }^{1}$

Assessment: The key outcomes of assessment provide measures of the burden of various diseases on a community or group of people. For genetics, these are measures of the burden of genetic diseases and their variants on various communities. Surveillance, monitoring, and investigatory research are integral aspects of assessment.

Policy development: This is where standards for health practice in the clinical setting, in the community, and throughout society are developed. As such, policy development is probably the most exciting part of public health. Success in policy development requires that public health be very active in listening to, informing, and educating the public; in developing specific standards and guidelines; and in promoting effective prevention and treatment services and high-quality health information.

Assurance: In public health, assurance addresses access to services, training, regulation, and evaluation. It is the job of public health to make sure that evaluation is always part of the development and implementation of health strategies. When applied to genetics, assurance plays a major role in seeing that genetic information is used appropriately. As discussed later in this paper, many efforts are currently under way to assure the best utilization of genetic testing and services in meeting the health needs of the population.

From the Office of Surgeon General, Department of Health and Human Services, Rockville, Maryland.

Allan S. Noonan, MD, MPH, Senior Advisor, Office of Surgeon General, Department of Health and Human Services, 5600 Fishers Lane, Rockville, MD 20857.

DOI: 10.1097/01.GIM.0000043818.54200.4F
Training has traditionally been a major role of public health. There are specific agencies within the United States public health system that focus on the training of individuals who practice throughout the health system, including people who provide clinical services and those responsible for preventive services. One challenging role of public health is attempting to assure that all client populations are represented among all health provider groups.

Public health is always wrestling with two types of factors: those that increase and those that decrease risk of disease. Among the factors most contributory to increased risk of premature morbidity and mortality in the United States are smoking, violence, lack of physical activity, inappropriate diet, and high-risk sexual behaviors. ${ }^{2}$ Public health attempts to reduce risk in areas such as sanitation, immunization, and workplace safety. By focusing on all these factors, public health brings the best science to the improvement of health and prevention of disease.

Genetics is but one piece of the puzzle of health determinants. The Human Genome Project has been a very exciting development for all of health. The responsibility of public health to understand the relationships between genetic variations and disease risk has grown. Public health must not only praise the sequencing of the genome, it must also extract and promote the use of this new information to reduce morbidity and mortality.

\section{Assessing genetic testing}

Since public health focuses on health outcomes, its first challenge is to look at the appropriateness and utilization of genetic tests, of which there are now more than $700 .^{3}$ The association of the various genotypes and gene variants with specific health outcomes must be better understood. This means that a great deal of scientific information must be documented before the 
question, How do we get from genotype to health outcome? can be answered.

First, gene-environment interactions should be analyzed. Environmental factors with direct genetic interactions have been documented for many diseases. Public health, therefore, must continue to assess environmental risk factors and develop strategies to prevent their detrimental impact.

The question of the appropriateness of genetic testing requires knowledge of the prevalence of the targeted disease, the association of the genetic variants of the disease, the penetrance and gene expression, and the interaction of the variants with any other known risk factors for the disease.

Data pertaining to ethical, legal, and social issues (ELSI) ${ }^{4}$; privacy and fairness; the integration of genetic technologies into health care; risk/benefit cost factors; and public and professional education must also be addressed before the utility of a genetic test can be determined. Public health must be able to assure the public that utilizing the tests is in its best interest.

\section{Centers for Disease Control and Prevention}

At the Centers for Disease Control and Prevention (CDC), the many genetic activities come under the purview of the Office of Genomics and Disease Prevention (OGDP). ${ }^{5}$ For instance, with regard to assessing genetic testing, the OGDP has a project under way with the Foundation for Blood Research (FBR). ${ }^{6}$ The ACCE project, ${ }^{7}$ which takes its name from the four components of evaluation-analytical validity, clinical validity, clinical utility, and the previously mentioned ELSI-is looking at all the variables. The aim of ACCE is to develop and test a model system to assess the availability and usefulness of existing data on DNA-based tests and testing algorithms. With this as the project's aim, the FBR has developed a set of questions regarding the analytical and clinical validity of tests. Questions assessing analytical validity include the following:

1. How often is a test positive when a mutation is present?

2. How often is it negative when a mutation is not present?

3. Is an internal quality control program defined and externally monitored?

4. How often does the test fail to give a usable result?

Questions assessing clinical validity include the following:

5. How often is the test positive when the disorder is present?

6. How often is the test negative when the disorder is not present?

7. What is the prevalence of the disorder in this setting?

8. What are the genotype-phenotype relationships?

Questions assessing the analysis of the clinical utility of a test include the following:

9. What is the natural history of the disorder?

10. Is there an effective remedy or acceptable action?

11. What are the results of pilot trials?
12. What are the financial costs and benefits associated with testing?

13. What educational materials have been developed and validated?

By using ACCE, researchers hope to identify data sources; collect, analyze, and interpret results; identify gaps in knowledge; and disseminate information. The goal of this effort is to facilitate the appropriate transition of genetic tests from the investigational setting to the clinical and public health settings.

Other CDC pilot projects looking at the assessment of genetic testing, issues of who is being tested, why, where, and what test is being used, are also under way. And a number of pilot projects are focusing on aspects of screening, such as newborn screening and screening of other populations, and determining the value of screening based on disease prevalence. Results so far indicate that the majority of genetic tests used today are for rare, single-gene disorders. However, as more tests are being considered for newborn screening, and associations between genes and common diseases are being discovered, the impact of genetic testing on public health is likely to increase. ${ }^{8}$

Informed consent is another important area the CDC is investigating. Appropriate application of informed consent procedures is being studied under ELSI. ${ }^{9}$ For much of the genetic testing that goes on in the United States today, the obtaining of informed consent is far from ideal. For example, informed consent for newborn screening, the best example we currently have of routine genetic screening, is sometimes obtained under what are very trying conditions for the mother. As the Genome Project progresses, public health will have increasing responsibility to assure that informed consent is obtained in the most comfortable situations possible for the patients.

The CDC is monitoring the literature on genetic disease and genetic and environmental interactions. Genetic information is being integrated into the agency's many population survey instruments, and tremendous efforts are under way to disseminate the information that results from these efforts.

Applied research is being funded at several different levels. For instance, in the area of genetic health education, a study is under way that asks whether it is more effective to educate the family directly or to prepare the physician to do so in order to assure that the individual makes the most rational decisions about his or her genetic health. ${ }^{10}$ These kinds of issues have not yet been resolved. Hence, for every new genetic test, these issues need to be explored.

The CDC is conducting a study looking at the genetic and environmental interaction for atherosclerosis risk. ${ }^{10}$ In a population-based screening study, a cohort of children, thought to be at increased risk for type 1 diabetes, is being followed. Those who are found to be at increased risk are enrolled in trials to attempt prevention. Hence the question of whether or not type 1 diabetes can be prevented is part of crucial, ongoing genetics research.

These studies demonstrate that public health efforts in genetics have an impact at all levels-at the system, provider, family, and individual levels. 


\section{The challenges of integrating genomics into public health}

Despite these illustrations of programs and successes in genomics, systems are not yet in place for the best use of expertise in this area. ${ }^{11}$ For newborn screening, for instance, each state has its own set of tests. Georgia is one state looking into improving newborn screening strategies. ${ }^{12,13}$ Evaluation of the Georgia system includes the following: how the information is collected, how it is stored, how it is used, how people are informed, and what kind of long-term information is provided to those who have been tested.

There are maternal phenylketonuria projects under way to assess best practices in genetic information follow-up. This question is also a high priority for the management of hemoglobinopathies. Although the sickle-cell trait is by far the largest product of newborn screening in the United States, only 14 of the 47 states screening for hemoglobinopathies had appropriate follow-up in 1998. As more products of the human genome research are produced, there will be a growing mandate to address what should be done with data that are not clinically important at the moment of screening, but may be of future importance to the individual and may pose problems of confidentiality. Managing the use of hemoglobinopathy trait information will provide guidelines for handling many of the sensitive outputs of the Human Genome Project.

Knowledge of the epidemiology of various genetic diseases is growing. Through the cystic fibrosis patient registry, ${ }^{14}$ the CDC is analyzing the morbidity factors of this particular genetic disease. ${ }^{15}$ But this once again brings up the question of diseases where sensitive information can be helpful or misused. There was an attempt to build a patient registry for sicklecell disease 15 years ago, but because of confidentiality problems, it proved to be impossible at that time. Now it appears that a nongovernmental group, representative of the screened population, is the appropriate body to maintain such a registry. Without a doubt, the practical problems of dealing with genetic disease information are enormous.

Prior to the passage of the Orphan Drug Act of 1983, lack of profit for the development of drugs to treat rare diseasesmany of them genetic - was a major problem. The incentives promulgated in this Act, however, have made it possible for government and industry to work together to improve the availability of treatments for many rare diseases. ${ }^{16-18}$

\section{Genomics and the Health Resources and Services Administration}

In the US public health system, the Health Resources and Services Administration (HRSA) ${ }^{19}$ has a genetic diseases program with a mission to promote health through early identification of individuals at risk from heredity disorders. HRSA is also responsible for the development of genetic services, both preventive and treatment services, that are comprehensive, accessible, family-centered, and culturally competent. HRSA's genetic diseases program facilitates the development of the ge- netics aspects of the public health infrastructure while also dealing with the differences among the states and the differences among public and private health providers. A major task of this program is to educate the public about newborn screening and foster linkages to health systems appropriate for follow-up services where needed. Making the best use of newborn screening data remains a very complex issue at this time and a major challenge for US public health.

Since HRSA is responsible for educating the public and providers, not surprisingly, HRSA's well-established priority of promoting genetic literacy to the individual, the family, and providers has increased significantly with the unraveling of the humane genome. As well as the public and providers, we should not forget the importance of making knowledge of genetics and public health available and understandable to those who make the legislative decisions to fund specific programs.

There will, though, be little progress in the integration of genomics and proteomics into public health unless immediate attention is paid to the education of health professionals. There are many health practitioners in our country who have little knowledge of genetics, but who will play a major role in the provision of preventive and treatment services for people with genetic diseases.

Fragmentation of services presents another obstacle. For example, in the United States, a comprehensive set of hemophilia treatment centers focuses on this one disease. These centers, which are separate from both state health departments and community health centers, provide comprehensive services in hemophilia testing, counseling, and education. Realistically, finite resources will prevent the development of similar centers for every genetic disease. However, the need to plan for testing, counseling, education, and coordination of services will grow dramatically as genomics and proteomics grow.

There are several tiers of public health function in the United States. One way to build an infrastructure and attempt to meet imminent needs is the federal grant-making process. The genetic diseases program in maternal and child health in HRSA, for example, has grants that it allocates every year to the states so that they can develop their regional state and local genetics plans and programs. For instance, when the utility of newborn screening for hemoglobinopathies was discovered in the mid-1980s, HRSA elected to fund the states to develop new programs to implement newborn hemoglobinopathy screening. The research was done at the NIH, but to bring the benefit of the research to people, HRSA has developed strategies to foster implementation at the state and local level.

The integration of newborn screening with other points of early identification of children with genetic conditions is also a priority for HRSA's genetic disease program in the Bureau of Maternal and Child Health. ${ }^{20}$ Coordination of this program with the CDC programs described earlier and with NIH research programs is a major effort of the US Department of Health and Human Services (HHS). 


\section{Collaborative efforts}

Collaborative efforts are essential. And this is where the Offices of the Surgeon General, the Assistant Secretary for Health, and the Secretary of the HHS come in. ${ }^{21,22}$ These offices will play an increasingly important role in fostering the diagnosis of genetic diseases and implementing genetic services. Indeed, collaboration among HHS agencies is the watchword of these new sciences. The NIH and HRSA are collaborating on workshops to engage primary care physicians; and the Agency for Health Resources and Quality (AHRQ), the NIH, and HRSA are working together on faculty development. ${ }^{23}$

Developing comprehensive genomics and proteomics capability is a far-reaching and demanding task. Without appropriate leadership, it would be impossible to pull all of these efforts together. In his support of newborn screening, Tommy G. Thompson, Secretary of the HHS, demonstrates this leadership.

Newborn screening is one of the most important expansions of child health services in the last 30 years. By using effective screening programs to identify babies with inheritable disorders, or at risk of developing them, we can diagnose newborns with treatable ailments earlier and help parents get them to the right specialists quickly. (Tommy G. Thompson, June 6, 2001)

Secretary Thompson is a former state governor who understands the importance of genetics in public health. With this level of support and with the kind of collaboration discussed above, genomics and proteomics are being successfully integrated into US public health.

To accomplish the necessary collaboration, there must be an active infrastructure that involves consumers, public health leaders, and genetics experts. In the United States we have learned much from the Council of Regional Networks for Genetic Services (CORN), an effort begun approximately 15 years ago. ${ }^{24}$ The effort focused on genetic diseases, realizing that although they impact the person on the street, many of the people who understand the realities of genetics are doctorally trained individuals who work in secluded settings. Based on an appreciation that programs to develop workable strategies could not be created without bringing these various interest groups together, a nationwide system of regional networks was formed that brought MDs and $\mathrm{PhD}$ geneticists together with public health officials and people from the community who had little formal training in genetics, health care, or health administration. Through their participation in CORN, these partners were able to share information, educate one another about strategies that would work in their settings, and set priorities for the spending of the limited resources available to their various communities. The networks played a major role in helping policymakers appreciate the importance of understanding genetics and genetic information. As a result of this effort, a mechanism-the National Newborn Screening and Genetics Resource Center (NNSGRC) — was recently put into place to facilitate making major changes in developing standards and guidelines for genetic diseases. ${ }^{25}$ Mechanisms such as NNSGRC will be key to the appropriate implementation of genomics and proteomics in the United States.

\section{Acknowledgments}

The author thanks his colleagues in the various government agencies whose work contributed to the content of this presentation. He especially thanks Francis S. Collins, Director of the National Human Genome Research Institute of the National Institutes of Health; Michele A. Puryear, Chief of Genetic Services of the Health Resources and Services Administration of the Department of Health and Human Services; and Paula W. Yoon, staff scientist at the Office of Genetics and Disease Prevention of the Centers for Disease Control and Prevention.

\section{References}

1. Institute of Medicine. The future of public health. Washington, DC: National Academy Press, 1988.

2. Mcginnis JM, Foege WH. Actual causes of death in the United States. JAMA 1993; 270:2207-2212.

3. GeneTests/GeneClinics, 2002. http://www.genetests.org/

4. US Department of Energy. Human Genome Project information: ethical, legal, and social issues (ELSI), 2002. http://www.ornl.gov/hgmis/elsi/elsi.html.

5. Centers for Disease Control and Prevention, Office of Genomics and Disease Prevention, 2002. http://www.cdc.gov/genomics/default.htm.

6. Foundation for Blood Research, 2002. http://www.fbr.org/

7. ACCE, a CDC-sponsored project carried out by the Foundation for Blood Research. Centers for Disease Control and Prevention, 2002. http://www.cdc.gov/genomics/ info/perspectives/files/testACCE.htm.

8. Yoon PW, Chen B, Faucett A, Cline M, Gwinn M, Lubin IM, Burke W, Khoury MJ. Public health impact of genetic tests at the end of the 20th century. Genet Med 2001;3:405-410.

9. Khoury MJ. Informed consent for population research involving genetics: a public health perspective. CDC Office of Genomics and Disease Prevention, 2002. http:// www.cdc.gov/genomics/info/reports/policy/editorial.htm.

10. Centers for Disease Control and Prevention, 2002. http://www.cdc.gov/genomics/ about/99res_proj.htm.

11. Beskow LM, Khoury MJ, Baker TG, Thrasher JF. The integration of genomics into public health research, policy, and practice in the United States. CDC Office of Genomics and Disease Prevention, 2002. http://www.cdc.gov/genomics/infi/reports/research/wheel.htm.

12. Centers for Disease Control and Prevention. Evaluating newborn screening program data systems-Georgia, 1998. Morb Mortal Wkly Rep 1999;48:1101-1104.

13. Georgia State, Division of Public Health, 2002. http://www.ph.dhr.state.ga.us/programs/genetics/index.shtml.

14. Goss $\mathrm{CH}$, Parker HW. CF patient registry serves as a vital resource to improved clinical care. Homeline 2002, http:/www.cff.org/images/customcontent/HLFeb02GA.pdf.

15. Wang SS, FitzSimmons SC, O'Leary LA, Rock MJ, Gwinn ML, Khoury MJ. Early diagnosis of cystic fibrosis in the newborn period and risk of Pseudomonas aeruginosa acquisition in the first 10 years of life: a registry-based longitudinal study. Pediatrics 2001;107:274-279.

16. Asbury CH. The orphan drug act: the first 7 years. JAMA 1991;265:893-897.

17. Haffner ME. Orphan drug product regulation-United States. Int J Clin Pharmacol Ther 2002; 40:84-88.

18. Peabody JW, Ruby A, Cannon P. The economics of orphan drug policy in the US: can the legislation be improved? Pharmacoeconomics 1995;8:374-384.

19. Health Resources and Services Administration, 2002. http://hrsa.gov/

20. HRSA Maternal and Child Health Bureau, 2002. http://mchb.hrsa.gov/programs/ default.htm.

21. US Office of the Surgeon General, 2002. http://www.suggeongeneral.gov/sgoffice.htm.

22. Secretary of the US Department of Health and Human Services, 2002. http://www.hhs.gov/

23. Genetics in Primary Care. HRSA/NIH/AHRQ, 2002. http://bhpr.hrsa.gov/medicine-dentistry/genepc.html.

24. Council of Regional Networks for Genetic Services. Link to archived CORN site, 2002. http://www.emory.edu/PEDIATRICS/corn/corn.htm.

25. HRSA National Newborn Screening and Genetics Resource Center, 2002. http:// genes-r-us.uth.scsa.edu/about.htm. 